\title{
Characterizing fish communities associated with drifting fish aggregating devices (FADs) in the Western Indian Ocean using underwater visual surveys
}

\author{
Marc Taquet ${ }^{1, \mathrm{a}}$, Gorka Sancho ${ }^{2}$, Laurent Dagorn ${ }^{3}$, Jean-Claude Gaertner $^{4}$, David Itano ${ }^{5}$, \\ Riaz Aumeeruddy ${ }^{6}$, Bertrand Wendling ${ }^{6}$ and Christophe Peignon ${ }^{7}$ \\ IFREMER, CRH, Av. Jean Monnet, BP 171, 34203 Sète Cedex, France \\ 2 College of Charleston, Grice Marine Laboratory, 205 Fort Johnson Road, Charleston, SC 29412, USA \\ 3 IRD, UR Thetis, CRH, Av. Jean Monnet, BP 171, 34203 Sète Cedex, France \\ 4 Centre d'Océanologie de Marseille, LMGEM, UMR CNRS 6117, rue de la Batterie des Lions, 13007 Marseille, France \\ 5 University of Hawaii, JIMAR, Pelagic Fisheries Research Program, 1000 Pope Road MSB 312 Honolulu, HI 96822, USA \\ ${ }^{6}$ Seychelles Fishing Authority, PO Box 449, Victoria, Mahe, Seychelles \\ 7 IRD, UR 109 Thetis, PO Box 570, Victoria, Mahe Seychelles
}

Received 21 September 2007; Accepted 17 December 2007

\begin{abstract}
We adapted a visual census method, mainly used in demersal and reef fish studies, to characterize fish communities associated to drifting fish aggregating devices (FADs) in the Western Indian Ocean. Drifting FAD associated fishes from both equatorial (Seychelles) and tropical waters (Reunion Island) were examined by divers. A total of 32 species (belonging to 16 families) were observed associated with drifting FADs in equatorial waters, and 24 species (14 families) were found around FADs in tropical waters. Twenty species were found in both regions. The highest number of species observed at a single FAD was $18(12 \pm 2$, mean $\pm \mathrm{SD})$ in equatorial and $13(10 \pm 3)$ in tropical waters, not counting circumnatant species loosely associated with the FAD. Some species like Kyphosus vaigiensis, Canthidermis maculata, Elagatis bipinnulata, Acanthocybium solandri and Coryphaena hippurus were observed on all or most of the surveys. In this study, the contribution in biomass of the 18 common species associated with drifting FADs (but excluding circumnatant species), represents more than $98 \%$ of the biomass. The overall biomass values of closely associated species remains well below tuna biomass estimates for circumnatant tuna schools at FADs, estimated as high as 200 tons. The species that most significantly contribute to the by-catch in tuna purse-seines logically match those that showing the highest biomass values in our surveys (Carcharhinus spp., Elagatis bipinnulata, Coryphaena hippurus, Canthidermis maculata, and Acanthocybium solandri). One of the most abundant and ubiquitous species in our study was the spotted oceanic triggerfish Canthidermis maculata that sometimes formed massive schools of many thousands individuals around the drifting FADs. Future research is needed to explore the role of such non tuna species in the attraction and aggregation processes of tuna around drifting FADs.
\end{abstract}

Key words: Pelagic fishes / Drifting FAD / Visual census / Fish aggregations / Seychelles / Reunion Island / Indian Ocean

Résumé - Caractéristiques des communautés de poissons associées aux DCP (dispositifs de concentration de poissons) observés en plongée dans l'ouest de l'océan Indien. Nous avons adapté une méthode d'observation en plongée sous-marine, surtout utilisée dans les études des poissons démersaux et des poissons récifaux, afin de caractériser les communautés de poissons associées aux DCP dérivants (dispositifs de concentration de poissons) dans une zone équatoriale (Seychelles) et dans une zone tropicale (île de La Réunion) situées dans l'ouest de l'océan Indien. Dans la zone équatoriale, 32 espèces (appartenant à 16 familles) ont été observées autour de ces dispositifs dérivants, contre 24 espèces (14 familles) dans la zone tropicale. Au total, 20 espèces sont communes aux deux zones. Le plus grand nombre d'espèces observées sous un DCP est de 18 (12 \pm 2 , moyenne \pm écart-type) en zone équatoriale, et 13 (10 \pm 3) en zone tropicale, sans tenir compte des espèces «circumnatantes » comme les thons qui ont un très grand rayon d'agrégation autour des DCP. Quelques espèces telles Kyphosus vaigiensis, Canthidermis maculata, Elagatis bipinnulata, Acanthocybium solandri et Coryphaena hippurus ont été observées lors de toutes les plongées. Les 18 espèces les

a Corresponding author: Marc. Taquet@ifremer.fr 
plus communément associées aux DCP dérivants (à l'exclusion des espèces «circumnatantes ») représentent plus de $98 \%$ de la biomasse. Néanmoins, la biomasse totale des espèces fortement liées aux DCP reste bien inférieure à celle estimée pour les thons qui peut dépasser 200 tonnes sous un seul DCP. Les espèces contribuant, de façon significative, aux captures accessoires des thoniers-senneurs correspondent à celles présentant, lors de nos plongées, les plus fortes biomasses (Carcharhinus spp., Elagatis bipinnulata, Coryphaena hippurus, Canthidermis maculata, et Acanthocybium solandri). Une des espèces les plus abondantes et ubiquistes, le baliste océanique, Canthidermis maculata, forme des bancs compacts de milliers d'individus autour des DCP dérivants. De futures investigations sont nécessaires pour explorer le rôle de ces espèces dans les processus d'attraction et d'agrégation des thons autour des DCP dérivants.

\section{Introduction}

Humans have exploited the strong association between certain fishes and floating objects throughout history (Jones 1722; Morales-Nin et al. 2000). Certain tropical tunas such as skipjack (Katsuwonus pelamis), yellowfin (Thunnus albacares) and bigeye tuna (Thunnus obesus) are commonly found forming large aggregations around floating objects and are the target of large-scale industrial fisheries (Fonteneau et al. 2000) in all oceans. Initially, most of these fisheries took advantage of the existence of natural floating logs, but soon fishermen started constructing artificial floating objects as fish aggregating devices (FADs) in order to aggregate schools of tuna and facilitate their capture. Currently, fishermen deploy thousands of FADs in the oceans (Moreno et al. 2007) and the majority of the tuna catches by industrial purse-seine vessels worldwide are made in aggregations under FADs (Fonteneau et al. 2000; Lennert-Cody and Hall 2000). However, tuna are not the only species found around FADs in the ocean. More than 333 species of fishes (pelagic and demersal) have been described to associate with floating objects in open waters (Castro et al. 2002), from small juvenile to large adult individuals. However, this represents an exhaustive list of all species that have been observed at least once, and it does not necessarily reflect the exact characteristics of common fish aggregations around drifting FADs.

The main objective of our paper is to provide the first description of the fish communities around drifting FADs using fishery-independent methods. This knowledge is necessary in order to use FADs as observatories of fish diversity in pelagic ecosystems. Romanov (2002) provided a first description of these fish communities found around drifting FADs in the Indian Ocean through the analysis of by-catch data collected by scientific observers onboard commercial purse seine vessels. However, such type of studies based on fisheries data might suffer from two weaknesses: (i) the selectivity of the fishing gear, and (ii) the fact that observers usually do not report abundance estimates, but only species composition. To avoid this issue, we used visual surveys by divers to directly observe the fish communities around FADs. This technique is largely used to study the biodiversity of reef fish communities (Harmelin-Vivien et al. 1985; Graham et al. 2006; Colvocoresses and Acosta 2007; Lecchini et al. 2007). Visual surveys are rarely used in pelagic waters, though they have been used around anchored FADs (D'Anna et al. 1999; Andalorro et al. 2002; Sinopoli et al. 2006) and oil platforms (Fabi et al. 2000; Fabi et al. 2002). We adapted the "stationary point-count" method used in the demersal fish visual census (Harmelin-Vivien et al. 1985; Bohnsack and Bannerot 1986;

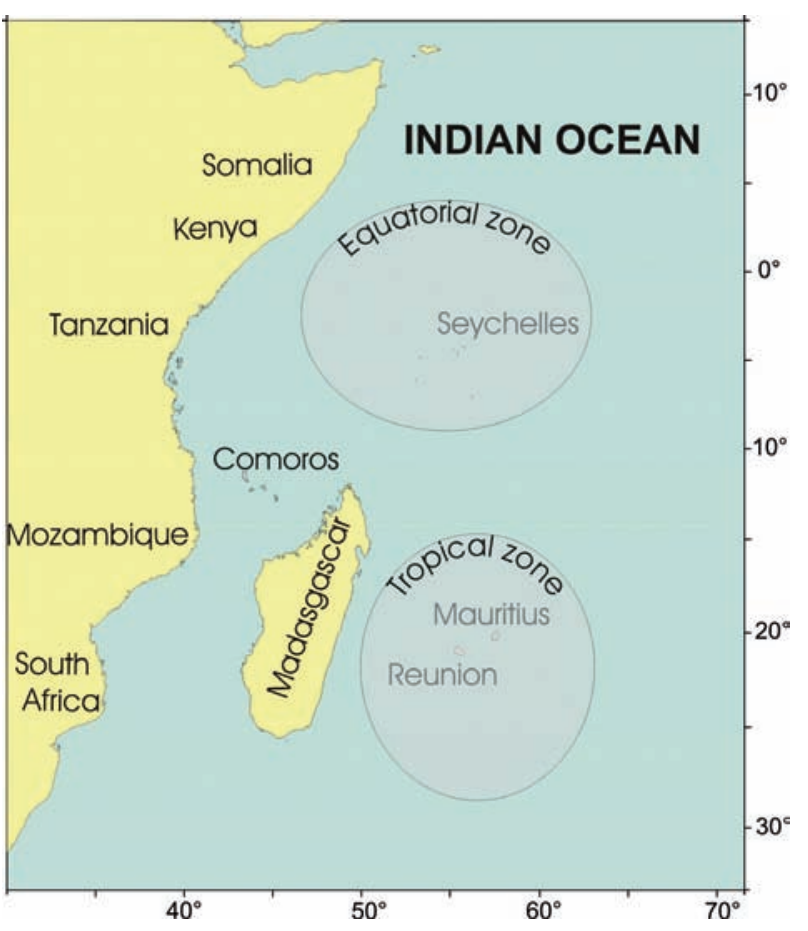

Fig. 1. Map of drifting FAD locations where fish censuses were done around Seychelles and Reunion Islands.

Jennings et al. 1995; Graham et al. 2006) to survey fish communities aggregated around drifting FADs. We also compared the characteristics of fish aggregations around drifting FADs in two different regions of the Western Indian Ocean (equatorial versus tropical waters).

\section{Material and methods}

\subsection{Studied areas and FADs}

Visual surveys of FADs were opportunistically made in two distinct areas of the Western Indian Ocean: a tropical area south of Reunion Island, studied between 2001 and 2003 in the frame of the DORADE project, and an equatorial zone around the Seychelles, investigated from 2003 to 2005 (Fig. 1) during the FADIO European project. The tropical area is not a drifting FAD fishing zone while the equatorial area is located in the middle of the fishing grounds of the European tuna purse seine fleet. A total of 11 FADs were deployed for this study and observed in the tropical area, and 22 FADS operated by commercial tuna seiners were observed in the equatorial area. Among 
those 33 FADs, only 4 of them had no underwater materials hanging underneath them (natural logs and ropes), while 29 had some underwater material attached to them (old netting panels for the equatorial FADs and large rice bags for tropical FADs). Among the 29 FADs with underwater material, 26 of them were bamboo rafts and 3 of theme consisted of underwater plastic drums with trailing netting panels. The exact age of the FADs in equatorial was not known, except for the 10 artificial FADs of the tropical area, as these ones were deployed by the scientific team. Visual surveys on the FADs in the tropical area were performed between 5 and 36 days after their initial deployment $(15.4 \pm 9.0$, mean \pm SD $)$.

\subsection{Visual census}

Visual censuses were performed only in clear waters with at least $25 \mathrm{~m}$ of horizontal visibility for security reasons, mainly to avoid possible shark attacks. Dives were performed by three experienced divers equipped with SCUBA, who always stayed close to each others to reduce disturbances to the fish communities. One of the 3 divers noted all the species encountered with their abundances and their estimated sizes on an underwater plastic sheet (the same diver performed all the visual surveys reported in this study). The second diver recorded on video all the species and schools observed during the survey and the third diver was in charge of the safety of the group.

Upon immediate arrival in the vicinity of a drifting FAD, the three divers would quickly enter the water in order to conduct a census of fishes in surface waters, as some elusive species like Lobotes surinamensis (Lobotidae) might depart the floating object shortly after the vessel arrived. An initial scan of the surface waters surrounding the FAD (minimum horizontal radius of $25 \mathrm{~m}$, i.e. the visual range in these waters) was made to identify and count fishes associated with the FAD in the first $10 \mathrm{~m}$ below the surface. Divers would then descend to a depth of $15 \mathrm{~m}$ below the FAD and survey deeper waters below the FAD down to approximately $30 \mathrm{~m}$ of depth (Fig. 2). This method is comparable to stationary pointcount technique commonly used in demersal and reef visual censuses. Nevertheless, some quick excursions to deeper waters or away from the FAD were sometimes necessary in order to verify the identification of particular fishes or schools. The depths of the divers ( 0 to $15 \mathrm{~m}$ ) and the visibility of the waters (approximately $25 \mathrm{~m}$ ) defined the semi-spherical volume of water centered on the FADs (horizontal radius of $25 \mathrm{~m}$ and a vertical radius of $30 \mathrm{~m}$ ).

Our main objective being the exhaustive detection of all fish species associated to a drifting FAD, our visual censuses ranged between 30 and 60 min (duration), depending on the amount of fishes present and the environmental conditions (see Graham et al. 2006 for an illustration of this strategy). Direct abundance estimates of individuals by species were conducted when fish schools consisted of less than 50 individuals, while more abundant species were estimated by assigning them to abundance classes. Each underwater visual census carried out by the main diver was complemented by a recording with an underwater video camera. The diver with the video camera

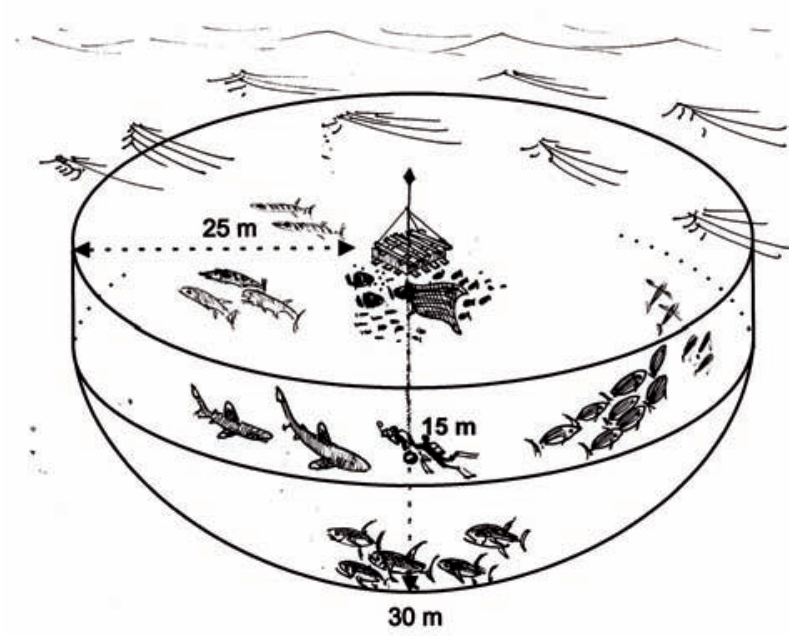

Fig. 2. Visual census method (1) divers scan of the surface waters and in the first $10 \mathrm{~m}$ below the surface, and (2) then at $15 \mathrm{~m}$ deep to survey fish species down to $30 \mathrm{~m}$ around fish aggregating device (FAD).

would first record each fish species found in close aggregation with the FAD, and then from a stationary point, $15 \mathrm{~m}$ below the FAD would slowly spin around $360^{\circ}$ and film all the fishes swimming around that position. The video camera was equipped with a wide angle lens to better estimate the abundance of schooling species. Videos were immediately viewed after each survey to verify the species lists and refine abundance estimates made underwater by surveying diver. The ecological and trophic characterizations of the species (planktivores, piscivores, omnivores and herbivore/planktivores) were selected on the basis of a large scale Indian Ocean ichthyologic study (Taquet and Diringer 2007). Divers also noted the behaviour of some species to determine if some species were using the underwater structures provided by FADs as a refuge against predators.

The fish species associated to FADs and logs were classified into 3 groups (Table 1) according to their distance to the floating object, as defined by Fréon and Dagorn (2000). Parin and Fedoryako (1999) subdivided the fish community around FADs into "intranatant species", which remain within $0.5 \mathrm{~m}$ of the floating object, "extranatant species" $(0.5-2 \mathrm{~m})$ and "circumnatant species" (+2 m), which are loosely associated with the object. Fréon and Dagorn (2000) adopted the same classification, but proposed different distances: $2 \mathrm{~m}$ for the intranatant/extranatant species, and up to $10-50 \mathrm{~m}$ for the extranatant/circumnatant species, considering that there is a substantial overlap between the distributions of these three communities. Some examples of these categories include the "intranatant" sergeant major damselfish (Abudefduf $\mathrm{sp}$.), which use FADs to take shelter from predators; the "extranatant": white-spotted triggerfish (Canthidermis maculatus) which stay in the vicinity of the drifting FAD; and "circumnatant" tuna, like skipjack tuna (Katsuwonus pelamis), which exhibit a wide range of movements in the vicinity of FADs.

All fish species observed during a survey were noted to determine the species composition of the aggregation. However, visual abundance estimates of circumnatant species are difficult to perform due to their loose aggregation with the 
Table 1. Characteristics of the visual censuses.

\begin{tabular}{|c|c|c|c|c|c|c|c|c|c|}
\hline & FAD type & Date & Time & $\begin{array}{c}\text { Duratio } \\
(\text { min) }\end{array}$ & Lat. & Long. & $\begin{array}{c}\text { Number } \\
\text { of fish }\end{array}$ & $\begin{array}{c}\text { Number } \\
\text { of species }\end{array}$ & $\begin{array}{c}\text { Biomass } \\
(\mathrm{kg})\end{array}$ \\
\hline \multicolumn{10}{|c|}{ Equatorial area (Seychelles) } \\
\hline E01 & Bamboo raft with net & $15 / 09 / 03$ & $15: 30$ & 30 & $2^{\circ} 10 \mathrm{~S}$ & $52^{\circ} 30 \mathrm{E}$ & 3489 & 13 & 1541 \\
\hline E02 & & $17 / 09 / 03$ & $8: 40$ & 30 & $0^{\circ} 01 \mathrm{~N}$ & $49^{\circ} 48 \mathrm{E}$ & 7091 & 13 & 3914 \\
\hline E03 & & $20 / 09 / 03$ & $7: 30$ & 60 & $3^{\circ} 29 \mathrm{~S}$ & $56^{\circ} 50 \mathrm{E}$ & 1948 & 13 & 2419 \\
\hline E04 & & $07 / 02 / 04$ & $8: 50$ & 30 & $5^{\circ} 38 \mathrm{~S}$ & $55^{\circ} 39 \mathrm{E}$ & 1105 & 12 & 509 \\
\hline E05 & & $07 / 02 / 04$ & $11: 10$ & 30 & $5^{\circ} 33 \mathrm{~S}$ & $55^{\circ} 54 \mathrm{E}$ & 339 & 13 & 178 \\
\hline E06 & & $07 / 02 / 04$ & $13: 20$ & 30 & $5^{\circ} 29 \mathrm{~S}$ & $55^{\circ} 57 \mathrm{E}$ & 1603 & 12 & 187 \\
\hline E07 & & 08/02/04 & $8: 10$ & 60 & $6^{\circ} 46 \mathrm{~S}$ & $55^{\circ} 06 \mathrm{E}$ & 2629 & 18 & 2545 \\
\hline E08 & Natural log & $11 / 02 / 04$ & $11: 45$ & 35 & $9^{\circ} 06 \mathrm{~S}$ & $56^{\circ} 08 \mathrm{E}$ & 3270 & 12 & 837 \\
\hline E09 & & $14 / 02 / 04$ & $14: 15$ & 30 & $6^{\circ} 49 \mathrm{~S}$ & $57^{\circ} 58 \mathrm{E}$ & 2332 & 9 & 1987 \\
\hline E10 & Spanish Drum FAD & $04 / 10 / 04$ & $10: 00$ & 40 & $3^{\circ} 36 \mathrm{~S}$ & $57^{\circ} 02 \mathrm{E}$ & 567 & 16 & 989 \\
\hline E11 & & $06 / 10 / 04$ & $13: 30$ & 40 & $3^{\circ} 02 \mathrm{~S}$ & $53^{\circ} 26 \mathrm{E}$ & 1629 & 12 & 473 \\
\hline E12 & + small logs & $12 / 10 / 04$ & $16: 00$ & 45 & $4^{\circ} 33 \mathrm{~S}$ & $53^{\circ} 25 \mathrm{E}$ & 111 & 7 & 37 \\
\hline E13 & Bamboo raft with net & $13 / 10 / 04$ & $9: 40$ & 60 & $5^{\circ} 15 \mathrm{~S}$ & $52^{\circ} 25 \mathrm{E}$ & 793 & 11 & 586 \\
\hline E14 & Big rope & $16 / 10 / 04$ & $14: 00$ & 40 & $4^{\circ} 04 \mathrm{~S}$ & $56^{\circ} 18 \mathrm{E}$ & 14153 & 12 & 10346 \\
\hline E15 & Bamboo raft with net & $03 / 02 / 05$ & $10: 50$ & 85 & $3^{\circ} 03 \mathrm{~S}$ & $55^{\circ} 03 \mathrm{E}$ & 2772 & 11 & 705 \\
\hline E16 & & $06 / 02 / 05$ & $10: 35$ & 70 & $2^{\circ} 18 \mathrm{~S}$ & $55^{\circ} 43 \mathrm{E}$ & 1013 & 14 & 1042 \\
\hline E17 & & $09 / 02 / 05$ & $10: 00$ & 90 & $3^{\circ} 58 \mathrm{~S}$ & $56^{\circ} 49 \mathrm{E}$ & 653 & 9 & 17 \\
\hline E18 & & $14 / 02 / 05$ & $9: 30$ & 60 & $3^{\circ} 18 \mathrm{~S}$ & $54^{\circ} 58 \mathrm{E}$ & 3198 & 13 & 1759 \\
\hline E19 & & $11 / 10 / 05$ & $8: 40$ & 60 & $5^{\circ} 20 \mathrm{~S}$ & $54^{\circ} 53 \mathrm{E}$ & 610 & 11 & 592 \\
\hline E20 & & $13 / 10 / 05$ & $8: 40$ & 50 & $3^{\circ} 12 \mathrm{~S}$ & $53^{\circ} 36 \mathrm{E}$ & 643 & 13 & 194 \\
\hline E21 & & $14 / 10 / 05$ & $13: 00$ & 45 & $3^{\circ} 05 \mathrm{~S}$ & $53^{\circ} 50 \mathrm{E}$ & 7765 & 14 & 533 \\
\hline E22 & & $18 / 10 / 05$ & $8: 50$ & 35 & $2^{\circ} 45 \mathrm{~S}$ & $55^{\circ} 04 \mathrm{E}$ & 1257 & 12 & 423 \\
\hline \multicolumn{10}{|c|}{ Tropical area (Réunion Island) } \\
\hline T01 & Experimental bamboo raft & $14 / 12 / 01$ & $8: 30$ & 120 & $22^{\circ} 05 \mathrm{~S}$ & $53^{\circ} 53 \mathrm{E}$ & 285 & 12 & 240 \\
\hline T02 & & $23 / 03 / 02$ & $8: 00$ & 120 & $26^{\circ} 38 \mathrm{~S}$ & $45^{\circ} 59 \mathrm{E}$ & 278 & 13 & 318 \\
\hline T03 & & $11 / 05 / 02$ & $16: 30$ & 45 & $20^{\circ} 45 \mathrm{~S}$ & $52^{\circ} 34 \mathrm{E}$ & 125 & 10 & 2507 \\
\hline T04 & & $11 / 05 / 02$ & 9:00 & 60 & $20^{\circ} 27 \mathrm{~S}$ & $52^{\circ} 16 \mathrm{E}$ & 95 & 13 & 2288 \\
\hline T05 & & $05 / 11 / 02$ & 11:00 & 45 & $23^{\circ} 49 \mathrm{~S}$ & $53^{\circ} 04 \mathrm{E}$ & 441 & 11 & 1006 \\
\hline T06 & & $22 / 05 / 03$ & $8: 30$ & 45 & $19^{\circ} 59 \mathrm{~S}$ & $53^{\circ} 02 \mathrm{E}$ & 149 & 13 & 173 \\
\hline Т07 & & $24 / 05 / 03$ & $8: 30$ & 60 & $20^{\circ} 22 \mathrm{~S}$ & $52^{\circ} 31 \mathrm{E}$ & 76 & 8 & 162 \\
\hline T08 & & $14 / 10 / 03$ & $9: 30$ & 30 & $23^{\circ} 38 \mathrm{~S}$ & $54^{\circ} 08 \mathrm{E}$ & 214 & 10 & 457 \\
\hline T09 & & $23 / 05 / 03$ & $11: 15$ & 45 & $20^{\circ} 22 \mathrm{~S}$ & $52^{\circ} 09 \mathrm{E}$ & 168 & 8 & 365 \\
\hline $\mathrm{T} 10$ & & $15 / 10 / 03$ & $9: 45$ & 75 & $21^{\circ} 38 \mathrm{~S}$ & $55^{\circ} 00 \mathrm{E}$ & 88 & 6 & 233 \\
\hline $\mathrm{T} 11$ & Big rope & $04 / 11 / 02$ & $17: 00$ & 60 & $24^{\circ} 16 \mathrm{~S}$ & $53^{\circ} 51 \mathrm{E}$ & 198 & 5 & 1239 \\
\hline
\end{tabular}

FAD. Circumnatant species such as yellowfin (Thunnus albacares), bigeye (Thunnus obesus), skipjack tuna (Katsuwonus pelamis) and blue marlin (Makaira nigricans) often swam outside the range of the visual observation by divers, precluding making accurate and repeatable estimates of abundance.

Minimum and maximum fish lengths of each species were estimated by trained divers (see Harmelin-Vivien et al. 1985, for details on the training method) with a minimum size set to $1 \mathrm{~cm}$ total length, and the average length was used to produce a biomass index (of all species except tuna). Due to the lack of precise Length/Weight relationships for several species, the same generic equation was used for all species:

$$
B_{\mathrm{i}}=10^{5} L_{\mathrm{i}}^{3} N_{\mathrm{i}}
$$

with $B_{\mathrm{i}}$ the biomass index $(\mathrm{kg}), L_{\mathrm{i}}$ the mean length of the species $i$ (in $\mathrm{cm}$ ) and $N_{\mathrm{i}}$ the number of fish counted for the species $i$.

\section{Results}

\subsection{Species composition}

A total of 32 species (5 intranatant, 23 extranatant and 4 circumnatant species) belonging to 16 families were observed associated with drifting FADs in equatorial waters (Table 2), and 24 species (4 intranatant, 18 extranatant and 2 circumnatant species) belonging to 14 families were found around FADs in tropical waters (Table 3 ). Twenty species were found in both regions, 12 species were exclusive to equatorial FADs and 4 were only observed at tropical ones. FADs in equatorial waters of the Indian Ocean had a slightly higher average number of species per FAD $(n=12.3 \pm 2.3)$ than Tropical FADs $(n=9.9 \pm 2.8)$ (Fig. 3a). A Wilcoxon rank sum test shows that these means are significantly different $\left(Z_{[22-11]}=2.096\right.$, $p=0.036)$. The highest number of species observed at a single FAD was 20 in equatorial and 13 in tropical waters, including circumnatant species. Some species were observed on all or most surveys: Kyphosus vaigiensis was found at all the FADs, Canthidermis maculata was found at $95.5 \%$ of equa- 
Table 2. Equatorial waters (Seychelles): list of species observed and associated with drifting FADS.

\begin{tabular}{|c|c|c|c|c|c|c|c|c|c|}
\hline Family & Species & Trophic classification & Ecological classification & $\begin{array}{c}\text { Fish } \\
\text { type(*) }\end{array}$ & $\begin{array}{c}\text { Number } \\
\text { of } \\
\text { fish }\end{array}$ & $\begin{array}{c}\% \\
\text { Concurrency }\end{array}$ & $\begin{array}{c}\% \\
\text { Biomass } \\
\text { Index }\end{array}$ & $\begin{array}{c}\text { Average } \\
\text { number of } \\
\text { fish per FAD }\end{array}$ & $\begin{array}{c}\text { Max. fish } \\
\text { per FAD }\end{array}$ \\
\hline Balistidae & Canthidermis maculata & Omnivore & Offshore pelagic & Ex & 16272 & $95.5 \%$ & $15.46 \%$ & 739.6 & 5000 \\
\hline Belonidae & Tylosurus crocodilus & Piscivore & Costal pelagic & Ex & 14 & $9.1 \%$ & $0.55 \%$ & 0.6 & 8 \\
\hline Carangidae & Carangoides fulvoguttatus & Piscivore & Costal pelagic & Ex & 2 & $9.1 \%$ & $0.00 \%$ & 0.1 & 1 \\
\hline Carangidae & Carangoides orthogrammus & Piscivore & Costal pelagic & Ex & 32 & $22.7 \%$ & $0.01 \%$ & 1.5 & 20 \\
\hline Carangidae & Caranx sexfasciatus & Piscivore & Costal \& Offshore pelagic & Ex & 1140 & $63.6 \%$ & $0.04 \%$ & 51.8 & 750 \\
\hline Carangidae & Decapterus macarellus & Planktonivore & Costal \& Offshore pelagic & Ex & 18101 & $72.7 \%$ & $4.33 \%$ & 822.8 & 7501 \\
\hline Carangidae & Elagatis bipinnulata & Piscivore & Costal \& Offshore pelagic & Ex & 13191 & $100.0 \%$ & $44.38 \%$ & 599.6 & 5000 \\
\hline Carangidae & Naucrates ductor & Omnivore & Costal \& Offshore pelagic & Ex & 58 & $45.5 \%$ & $0.02 \%$ & 2.6 & 15 \\
\hline Carangidae & Seriola lalandi & Piscivore & Costal \& Offshore pelagic & Ex & 46 & $22.7 \%$ & $0.03 \%$ & 2.19 & 30 \\
\hline Carangidae & Seriola rivoliana & Piscivore & Costal \& Offshore pelagic & Ex & 168 & $86.4 \%$ & $0.12 \%$ & 7.6 & 30 \\
\hline Carangidae & Uraspis helvola & Piscivore & Demersal \& Offshore pelagic & Ex & 199 & $54.5 \%$ & $0.16 \%$ & 9.1 & 50 \\
\hline Carcharhinidae & Carcharhinus falciformis & Piscivore & Offshore pelagic & Ex & 209 & $77.3 \%$ & $12.01 \%$ & 9.5 & 100 \\
\hline Carcharhinidae & Carcharhinus longimanus & Piscivore & Offshore pelagic & Ex & 1 & $4.5 \%$ & $1.35 \%$ & 0.1 & 1 \\
\hline Coryphaenidae & Coryphaena hippurus & Piscivore & Offshore pelagic & Ex & 643 & $95.5 \%$ & $13.30 \%$ & 29.2 & 100 \\
\hline Ephippidae & Platax teira & Omnivore & Reef & Ex & 10 & $18.2 \%$ & $0.00 \%$ & 0.5 & 7 \\
\hline Kyphosidae & Kyphosus vaigiensis & Herbivore - Planktonivore & Demersal \& Costal \& Offshore pelagic & Ex & 7435 & $100.0 \%$ & $1.54 \%$ & 337.9 & 3000 \\
\hline Lobotidae & Lobotes surinamensis & Piscivore & Offshore pelagic & Ex & 31 & $45.5 \%$ & $0.11 \%$ & 1.4 & 6 \\
\hline Monacanthidae & Acreichthys sp. & Herbivore - Planktonivore & Reef & In & 14 & $18.2 \%$ & $0.00 \%$ & 0.6 & 5 \\
\hline Monacanthidae & Aluterus monoceros & Omnivore & Demersal \& Costal \& Offshore pelagic & Ex & 23 & $27.3 \%$ & $0.03 \%$ & 1.1 & 10 \\
\hline Monacanthidae & Aluterus scriptus & Omnivore & Demersal \& Costal \& Offshore pelagic & Ex & 46 & $18.2 \%$ & $0.07 \%$ & 2.1 & 40 \\
\hline Monacanthidae & Cantherhines sp. & Omnivore & Reef & In & 2 & $4.5 \%$ & $0.00 \%$ & 0.1 & 2 \\
\hline Nomeidae & Psenes cyanophrys & Omnivore & Offshore pelagic & Ex & 433 & $40.9 \%$ & $0.00 \%$ & 19.7 & 200 \\
\hline Pomacentridae & Abudefduf vaigiensis & Herbivore - Planktonivore & Reef & In & 633 & $86.4 \%$ & $0.00 \%$ & 28.8 & 100 \\
\hline Priacanthidae & Heteropriacanthus cruentatus & Piscivore & Reef & In & 1 & $4.5 \%$ & $0.00 \%$ & 0.1 & 1 \\
\hline Scombridae & Acanthocybium solandri & Piscivore & Costal \& Offshore pelagic & Ex & 184 & $72.7 \%$ & $5.34 \%$ & 8.4 & 100 \\
\hline Scombridae & Euthynnus affinis & Piscivore & Costal pelagic & Ex & 1 & $4.5 \%$ & $0.00 \%$ & 0.1 & 1 \\
\hline Sphyraenidae & Sphyraena barracuda & Piscivore & Costal \& Offshore pelagic & Ex & 60 & $22.7 \%$ & $1.15 \%$ & 2.7 & 30 \\
\hline Teraponidae & Terapon theraps & Omnivore & Reef & In & 22 & $9.1 \%$ & $0.00 \%$ & 1 & 20 \\
\hline Istiophoridae & Makaira nigricans & Piscivore & Offshore pelagic & Circum & 1 & $4.5 \%$ & - & 0.1 & 1 \\
\hline Scombridae & Katsuwonus pelamis & Piscivore & Offshore pelagic & Circum & 9000 & $18.2 \%$ & - & 409.1 & 4000 \\
\hline Scombridae & Thunnus albacares & Piscivore & Offshore pelagic & Circum & 23000 & $45.5 \%$ & - & 1044.2 & 10000 \\
\hline Scombridae & Thunnus obesus & Piscivore & Offshore pelagic & Circum & 2200 & $9.1 \%$ & - & 100 & 2000 \\
\hline
\end{tabular}

(*) Fish type: "Intranatant species" (In) remain within 0.5 m of the floating object; "Extranatant species" (Ex), within 0.5-2 m; "Circumnatant species" (Circum) are loosely associated with the object (after Fréon and Dagorn 2000). 
Table 3. Tropical waters (Reunion Island): list of species observed and associated with drifting FADs.

\begin{tabular}{|c|c|c|c|c|c|c|c|c|c|}
\hline Family & Species & Trophic classification & Ecological classification & $\begin{array}{c}\text { Fish } \\
\text { type(*) }\end{array}$ & $\begin{array}{c}\text { Number } \\
\text { of fish }\end{array}$ & $\begin{array}{c}\% \\
\text { Concurrency }\end{array}$ & $\begin{array}{c}\% \\
\text { Biomass } \\
\text { Index }\end{array}$ & $\begin{array}{c}\text { Average } \\
\text { number of } \\
\text { fish per FAD }\end{array}$ & $\begin{array}{l}\text { Max. fish } \\
\text { per FAD }\end{array}$ \\
\hline Balistidae & Canthidermis maculata & Omnivore & Offshore pelagic fish & Ex & 349 & $81.8 \%$ & $2.58 \%$ & 31.7 & 100 \\
\hline Carangidae & Carangoides orthogrammus & Piscivore & Costal pelagic fish & Ex & 5 & $9.1 \%$ & $0.04 \%$ & 0.5 & 5 \\
\hline Carangidae & Caranx sexfasciatus & Piscivore & Costal \& Offshore pelagic fish & Ex & 58 & $45.5 \%$ & $0.01 \%$ & 5.3 & 30 \\
\hline Carangidae & Decapterus macarellus & Planktonivore & Costal \& Offshore pelagic fish & Ex & 246 & $45.5 \%$ & $0.11 \%$ & 22.4 & 100 \\
\hline Carangidae & Elagatis bipinnulata & Piscivore & Costal \& Offshore pelagic fish & Ex & 82 & $72.7 \%$ & $1.79 \%$ & 7.5 & 41 \\
\hline Carangidae & Naucrates ductor & Omnivore & Costal \& Offshore pelagic fish & Ex & 11 & $18.2 \%$ & $0.00 \%$ & 1 & 10 \\
\hline Carangidae & Selar crumenophthalmus & Planktonivore & Costal pelagic fish & Ex & 3 & $9.1 \%$ & $0.00 \%$ & 0.3 & 3 \\
\hline Carangidae & Seriola rivoliana & Piscivore & Costal \& Offshore pelagic fish & Ex & 29 & $72.7 \%$ & $0.13 \%$ & 2.7 & 13 \\
\hline Carangidae & Uraspis helvola & Piscivore & Demersal \& Offshore pelagic fish & Ex & 9 & $27.3 \%$ & $0.12 \%$ & 0.8 & 4 \\
\hline Carcharhinidae & Carcharhinus falciformis & Piscivore & Offshore pelagic fish & Ex & 2 & $9.1 \%$ & $0.43 \%$ & 0.2 & 2 \\
\hline Coryphaenidae & Coryphaena hippurus & Piscivore & Offshore pelagic fish & Ex & 214 & $90.9 \%$ & $31.34 \%$ & 19.5 & 75 \\
\hline Diodontidae & Diodon histrix & Omnivore & Reef fish & In & 1 & $9.1 \%$ & $0.00 \%$ & 0.1 & 1 \\
\hline Kyphosidae & Kyphosus vaigiensis & Herbivore - Planktonivore & Demersal \& Costal \& Offshore pelagic fish & Ex & 555 & $100.0 \%$ & $0.79 \%$ & 50.5 & 105 \\
\hline Labridae & Labroides dimidiatus & Omnivore & Reef fish & In & 2 & $9.1 \%$ & $0.00 \%$ & 0.2 & 2 \\
\hline Lobotidae & Lobotes surinamensis & Piscivore & Offshore pelagic fish & Ex & 9 & $54.5 \%$ & $0.25 \%$ & 0.8 & 3 \\
\hline Monacanthidae & Aluterus monoceros & Omnivore & Demersal \& Costal \& Offshore pelagic fish & Ex & 12 & $27.3 \%$ & $0.15 \%$ & 1.19 & 9 \\
\hline Monacanthidae & Aluterus scriptus & Omnivore & Demersal \& Costal \& Offshore pelagic fish & Ex & 5 & $18.2 \%$ & $0.03 \%$ & 0.5 & 4 \\
\hline Monacanthidae & Pervagor melanocephalus & Herbivore - Planktonivore & Reef fish & In & 16 & $18.2 \%$ & $0.00 \%$ & 1.45 & 15 \\
\hline Nomeidae & Psenes cyanophrys & Omnivore & Offshore pelagic fish & Ex & 186 & $63.6 \%$ & $0.01 \%$ & 16.9 & 45 \\
\hline Pomacentridae & Abudefduf vaigiensis & Herbivore - Planktonivore & Reef fish & In & 31 & $63.6 \%$ & $0.00 \%$ & 2.8 & 16 \\
\hline Scombridae & Acanthocybium solandri & Piscivore & Offshore pelagic fish & Ex & 287 & $100.0 \%$ & $61.79 \%$ & 26.1 & 75 \\
\hline Sphyraenidae & Sphyraena barracuda & Piscivore & Costal \& Offshore pelagic fish & Ex & 2 & $18.2 \%$ & $0.44 \%$ & 0.2 & 1 \\
\hline Istiophoridae & Makaira nigricans & Piscivore & Offshore pelagic fish & Circum & 1 & $9.1 \%$ & - & 0.2 & 1 \\
\hline Scombridae & Thunnus albacares & Piscivore & Offshore pelagic fish & Circum & 1 & $9.1 \%$ & - & 0.2 & 1 \\
\hline
\end{tabular}

(*) Fish type: "Intranatant species" (In) remain within $0.5 \mathrm{~m}$ of the floating object; "Extranatant species" (Ex), within 0.5-2 m; "Circumnatant species" (Circum) are loosely associated with the object (after Fréon and Dagorn 2000). 


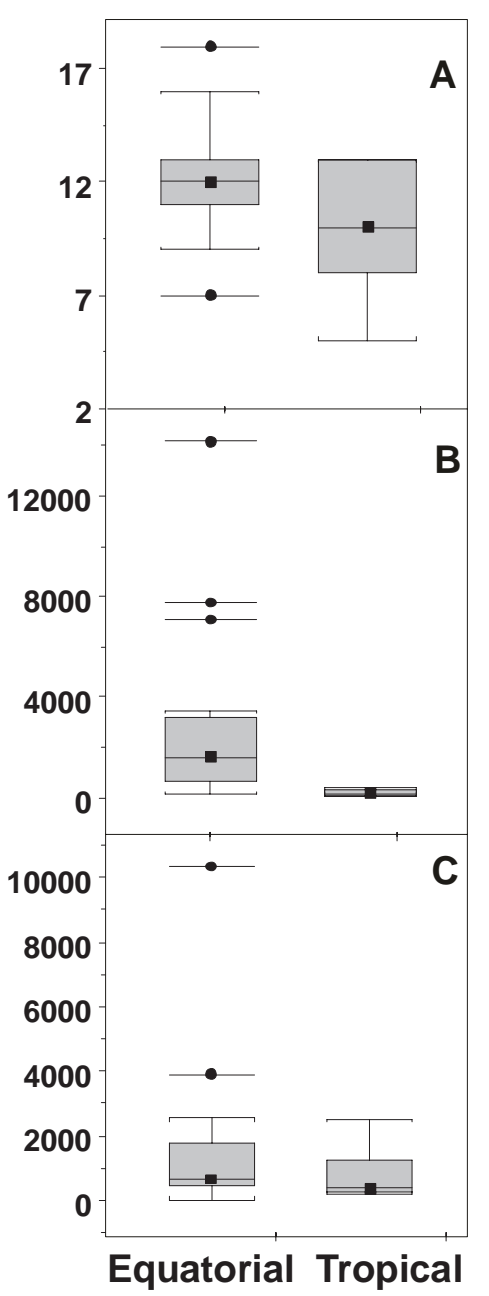

Fig. 3. Number of species (A), number of fishes (B) and estimated biomass $(\mathrm{C})$ per individual FAD in the equatorial and tropical regions. The boxes represent the quartiles around the median (line inside the box); the dots outside the box represent the outliers.

torial and $82 \%$ of tropical FADs, Elagatis bipinnulata was present at all equatorial FADs and $75 \%$ of tropical ones. Of the large predatory species observed, Acanthocybium solan$d r i$ were recorded at $73 \%$ of equatorial FADs and all tropical ones, Coryphaena hippurus was found at $95.5 \%$ of equatorial and $91 \%$ of tropical FADs, and Carcharhinus falciformis, was at $77.3 \%$ of equatorial FADs, but only at $7 \%$ of tropical FADs. Only one yellowfin tuna specimen (Thunnus albacares) was observed at a tropical FAD, while yellowfin tuna (Thunnus albacares) were observed at $45.5 \%$ of the equatorial FADs, sometime associated with two other species skipjack (Katsuwonus pelamis) at $18 \%$ and bigeye tuna (Thunnus obesus) at $9 \%$ of the studied FADs.

\subsection{Fish abundance}

The average number of aggregated fishes observed per FAD was higher at equatorial FADs $(2680 \pm 3245)$ than at tropical ones $(192 \pm 109)$ (Fig. 3b). Fish abundance was highly variable between individual FADs in the equatorial region

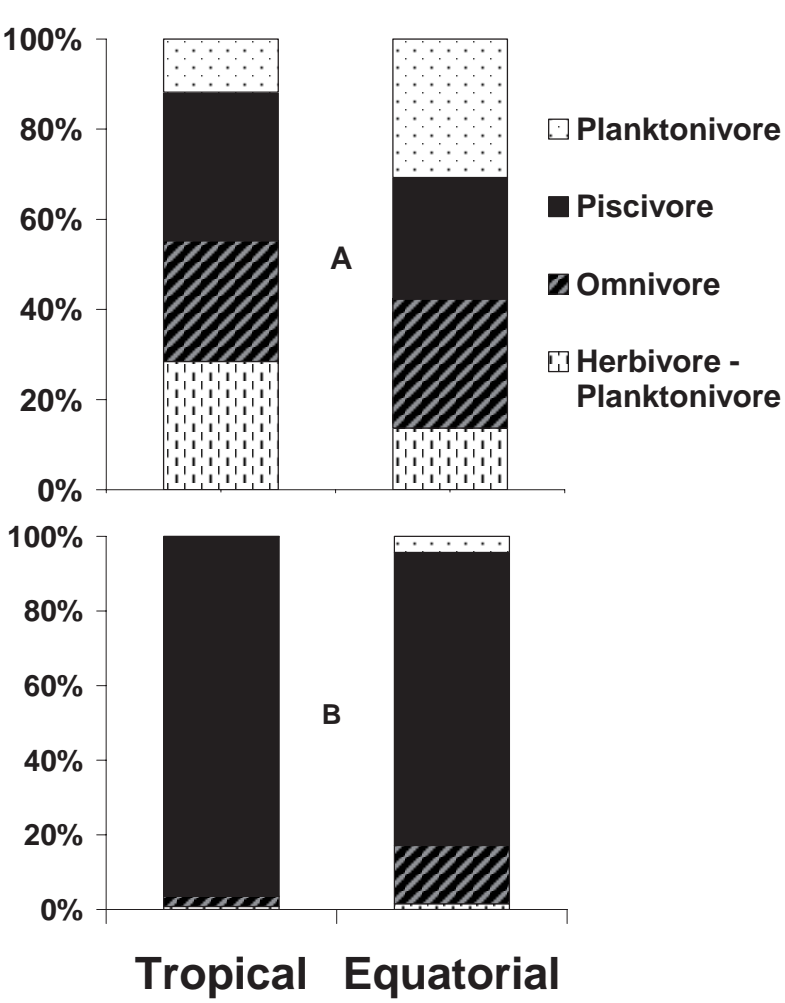

Fig. 4. Trophic classification of fishes associated with FADs shown as $\%$ of fish abundance (A) and biomass (B) for tropical and equatorial regions.

(minimum: 111, maximum: 14 531) (Fig. 3b). Abundance variability at tropical FADs was much lower (min: 76, max: 441 aggregated fishes). Overall the most abundant species in the equatorial FAD censuses were Decapterus macarellus, Canthidermis maculata and Elagatis bipinnulata, which, combined together, accounted for $80.7 \%$ of all the fishes observed (Table 2). The most abundant predatory species was Caranx sexfasciatus (juvenile stage, mean length $6.8 \mathrm{~cm}$ ), which was observed associated to $63.5 \%$ of the equatorial FADs and $45.5 \%$ of the tropical ones. At tropical FADs, Kyphosus vaigiensis was the most abundant species, followed by Canthidermis maculata (Table 3). A clear difference between both regions was the much higher abundance of silky sharks (Carcharhinus falciformis) at equatorial FADs (mean $9.5 \pm 21.4$ shark per FAD) than at tropical ones $(0.18 \pm$ 0.80 per FAD only).

\subsection{Fish biomass}

Considering the estimated average biomass of fishes associated with drifting FADs (excluding circumnatant species, which in the case of tuna can form huge schools under FADs), equatorial FADs held almost twice as much biomass per FAD $\left(B_{\mathrm{i}}=1446 \pm 2216 \mathrm{~kg}\right)$ as tropical ones $\left(B_{\mathrm{i}}=817 \pm 856 \mathrm{~kg}\right)$ (Fig. 3c), with variability also much higher in biomass estimates at individual FADs in the equatorial region. Minimum and maximum $B_{\mathrm{i}}$ values were $17 \mathrm{~kg}$ and $10346 \mathrm{~kg}$ respectively in equatorial waters but ranged only between $162 \mathrm{~kg}$ and 
$2288 \mathrm{~kg}$ in tropical waters. In the equatorial region, Elagatis bipinnulata was the largest contributor to the total biomass estimate (44.4\%), followed by Canthidermis maculata $(15.5 \%)$, Coryphaena hippurus (13.3\%) and Carcharhinus falciformis (12.0\%) (Table 2). Biomass estimates at tropical FADs were dominated by the large predatory Acanthocybium solandri (61.8\%) and Coryphaena hippurus (31.3\%) (Table 3). Finally, the contributions in biomass of the 18 common species (with the exception of circumnatant species) were very similar in the two regions with $99.99 \%$ and $98.07 \%$ for tropical and equatorial regions respectively. Such relative biomass estimates are well below tuna biomass that was estimated around the FADs through acoustic methods, with a maximum of 200 tons.

\subsection{Fish trophic categories}

The abundance of fishes assigned to different trophic categories was very homogeneous, with similar distributions of abundance values across all four trophic categories (planktivores, piscivores, omnivores and herbivore/planktivores) both in equatorial and tropical regions (Fig. 4a). Piscivores fishes clearly dominated drifting FAD associated communities in both regions when estimated biomass of fishes was employed to characterize the communities (Fig. 4b).

\subsection{Fish behavior}

All juvenile reef fishes (Abudefduf vaigiensis, Acreichthys sp., Cantherhines sp., Didon histrix, Heteropriacanthus cruentatus, Labroides dimidiatus, Platax teira, Pervagor melanocephalus, Terapon theraps) were observed to be constantly hiding in the underwater structures of FADs when divers approached, likely a behavior to avoid predation. Other species like Kyphosus vaigiensis or Canthidermis maculata were observed using two different strategies to avoid predators depending on their abundance. If the number of fishes was low (several individuals only), the FAD structure was used as a refuge, hiding among the netting or bamboo floats. But when found in high abundance, these species used schooling behaviors centered on the FAD in response to approaching predators such as Sphyraena barracuda. In situations where predators were observed attacking fishes, most fishes would swim very closely to the FAD, increasing the density of their aggregation.

\section{Discussion}

\subsection{A standardized visual census method}

A preliminary analysis of the data collected during the visual censuses showed that all the intranatant and extranatant species are detected within a 30 min period of survey. We recommend that each dive lasts at least $30 \mathrm{~min}$, which was found to be the minimum time to exhaustively determine and count fish at all FADs. Then, dives could last longer, depending on the number of species and fish around FADs, so that all intra- and extranatant species can be determined and counted.
We have shown, through 33 visual surveys, that our protocol is repeatable in a scientific manner, so that it can be used for monitoring many different FADs and conduct comparative analysis. However, if this technique is accurate for intra- and extranatant species, The detection of circumnatant species like tunas was not guaranteed, even with 60-min surveys due to the possible long excursions (more than one hour) performed by these species, often far off the FAD (Dagorn et al. 2007). In addition, time was not the only limitation factor for the census of circumnatant species, as swimming depth outside the visual range of the divers could also be a critical point for these species. So our results show that the visual census method is not accurate to observe the circumnatant species. Complementary approaches such as acoustic surveys are necessary to detect and estimate circumnatant species abundance around FADs, and should be incorporated to future diversity studies.

Effects of FAD characteristics. The sample size of our study was too low to investigate the possible effects of FAD characteristics (including time at sea) on fish aggregations. However, the physical characteristics of floating objects do not seem to play a role in the attraction of fishes (Fréon and Dagorn 2000). Observations on 5518 floating objects in the eastern Pacific Ocean, of which 2793 resulted in a fishing set by purse seiners, did not clearly indicate any particular characteristic (i.e., size, surface, volume, colour) that might explain different attractive effects (Hall et al. 1999). The only patterns observed were that very small and very large objects are less attractive than medium sized ones, and that black coloured objects seem to be more effective in attracting fishes. All of our floating objects had a similar size (medium size category when using Hall et al. 1999 definitions). However, we acknowledge that the presence of underwater structures such has hanging netting could affect the species composition, if some intranatant species cannot hide under floating objects with no hanging materials. Only 4 of our 33 samples floating objects did not have hanging materials, which precludes us from performing meaningful statistical comparisons, but fish aggregations around those floating objects showed similar number of species $(9.5 \pm 3.3)$ and higher fish abundances (4988 \pm 6243$)$ than the other FADs. Time at sea is likely to play a key role in the characteristics of fish aggregations. However, for intraand circumnatant species, it seems that the time needed to aggregate around FADs is quite short. By extracting the empirical knowledge of skippers of European purse seiners in the Western Indian Ocean, Moreno et al. (2007) determined that intra- and extranatant species usually need between 1 week and 2-3 weeks to colonize a FAD. All the FADs sampled in the equatorial area were more than one month old. The 10 artificial FADs of the tropical area were all sampled from 1 to 5 weeks after deployment. Therefore, we can consider that the time at sea might not be a significant factor in our study to explain possible differences between FADs. Further studies on the colonization processes around FADs are clearly needed.

\subsection{Species composition and fish behavior}

Most of the species observed in our study are caught as bycatch by the tuna purse-seine fleet in the equatorial Indian 
Ocean when fishing on floating objects (Romanov 2002). Our survey registered 32 fish species associated with drifting FADs in the same waters (equatorial area), while Romanov (2002) measured 42 fish taxa being captured in purse seines. The comparison between both studies is complicated by differences in taxonomic accuracy when identifying fishes. In our study, all fishes were identified at the species level, while in Romanov's study 28 of the 42 fish taxa were identified to the species level and the other 14 taxa were identified to the genus or family level. Nevertheless, we observed 4 circumnatant species versus 11 taxa ( 9 species and 2 genus level) recorded by Romanov (2002), and 28 intra- or extranatant species in our study versus 28 taxa (16 fully identified species and 12 genus level) by Romanov (2002). The fishery observers were more efficient in counting circumnatant species and deep species like Gempylidae (Ruvettus pretiosus) that are caught in the large purse seines, while divers could more easily identify and count extranatant species. Moreover, the use of divers allowed the observation of small juvenile reef fish species (intranatant species) that were not recorded by fishery observers due to the gear selectivity. The species that most significantly contribute to the by-catch in tuna purse-seines logically match those that showed the highest biomass values in our surveys (Carcharhinus spp., Elagatis bipinnulata, Coryphaena hippurus, Canthidermis maculata, and Acanthocybium solandri). While scientific observers onboard purse seine vessels can provide more samples than scientific divers on fish aggregations around drifting FADs, divers have the advantage of obtaining data independent of fisheries activities. This study clearly illustrates the advantage of diver surveys: it provides more accurate estimates of intra- and extranatant species (i.e. by-catch species of purse seiners) than fisheries observers, and they are fishery-independent.

Fish communities aggregated under drifting FADs in equatorial and tropical waters of the Western Indian Ocean had many species in common (20 species) mainly extranatant ones. These common species represent almost the total biomass ( $>98 \%$ ) of associated fish (excluding circumnatant species) in each region. Therefore FADs appear to be an efficient tool to observe and study these species that are usually very difficult to access due to their large and scattered geographical distribution in the tropical and subtropical offshore waters.

Juvenile reef fishes (e.g. Abudefduf vaigiensis, Diodon histrix, Cantherhines sp.) use FADs as a substitute habitat when they are unable to locate a suitable reef habitat following their pelagic larval stage (Castro et al. 2002). In our study, one of these species (Abudefduf vaigiensis) was much more abundant in equatorial waters than tropical ones. One possible explanation for this difference in abundance could be the proximity of the large Seychellois plateau, a potential source of larvae to FADs sampled in equatorial waters.

Our observations on fish behavior confirmed the use of underwater structures by most small species (all the intranatant species and most of the juvenile stages of extranatant species) to avoid predators (Parin and Fedoryako 1999). Some species, like Kyphosus vaigiensis or Canthidermis maculata, alternated schooling tactics and hiding behavior below FADs to avoid predators, depending on their local abundance. FADs appear to function as a physical refuge from predators for some species when other avoidance behavior more commonly used in open waters (i.e. schooling) are not efficient due to low abundances. Interestingly, FAD associated fish species (excluding small circumnatant Scombrid species) are very rarely found in stomach contents of tuna (Buckley and Miller 1994; Ménard et al. 2000), and do not dominate the diet of other abundant predators found in closer association with FADs such as adult Coryphaena hippurus (Oxenford 1999; Olson and Galvan-Magana 2002; Taquet 2004) and Acanthocybium solandri (Bochard and Techer 2004). The diet of piscivorous species that have high occurrence rates at FADs, but are not very abundant (i.e. Sphyraena barracuda and Carcharhinus falciformis) is not well described, and therefore the trophic importance of FAD aggregations cannot be established for these species. However, it is likely that the role of FADs for some species is mixed, providing both refuge from predation and a source of food, depending on the circumstances.

\subsection{Fish abundance and biomass}

While equatorial and tropical FADs showed somewhat different species composition, they greatly differed in their relative abundances. Equatorial aggregations often displayed large numbers of small species (Decapterus macarellus and Canthidermis maculata), which were present in tropical aggregations, but in much lower abundance.

The biomass in tropical FAD aggregations was dominated by two large predatory species, Acanthocybium solandri and Coryphaena hippurus, which accounted for $93 \%$ of the fish biomass. Equatorial FAD aggregation biomass (excluding tuna) was dominated by Elagatis bipinnulata, followed to a lesser degree by Canthidermis maculata, Coryphaena hippurus and Carcharhinus falciformis. Species like Acanthocybium solandri, Coryphaena hippurus and Elagatis bipinnulata formed the majority of the aggregated biomass closely associated to the FADs in both regions. If the biomass of non-tuna species is usually low as compared to tuna biomass (average of $2.7 \%$ from Romanov 2002, confirmed by this study), the diversity of species can be considered as relatively high. Such an index should be taken into account when assessing the effects of FAD fishing on pelagic ecosystems.

Nevertheless, the fishery status of non-tuna FAD associated species is very different, as some species are simply discarded by equatorial industrial purse seine fishery while they are target species in the tropical small scale fisheries around anchored FADs (e.g. Coryphaena hippurus). Therefore, it is necessary to study the interactions between large-scale and small-scale fisheries to examine if catches of large-scale fisheries impact catches of small-scale fisheries (Dempster and Taquet 2004).

\section{Future research}

Making observations on drifting FADs in high seas is extremely difficult and expensive. This study provides the very first data on the composition of the fish communities around drifting FADs, by using a standardized visual census method that allows to conduct repeatable observations. We have shown 
that the species composition and abundance varies between equatorial and tropical areas. Future studies should collect more data in different regions to examine the spatial, but also seasonal variability of fish aggregations around FADs. Such fishery-independent data could be used to monitor the biodiversity of the pelagic realm, which is very difficult to access. So far, only a few studies investigated the biodiversity of pelagic waters (e.g. Worm et al. 2003) and they were dependent on fisheries data. Innovative ways of observing the diversity of fishes in the open ocean must be developed in order to properly investigate the effects of climate change and anthropogenic pressure on these ecosystems. In the same way that visual census has been used to assess the diversity of reef fishes (Harmelin-Vivien et al. 1985; Graham et al. 2006; Colvocoresses and Acosta 2007; Lecchini et al. 2007), we argue that visual surveys around FADs, which act as our sampling tool in the open ocean, should be regularly conducted to assess the diversity of fishes in the pelagic waters.

Colonization processes of FADs should clearly be investigated in the future. What are the first species to colonize a FAD? When a purse seiner catches a fish aggregation around a FAD, it provokes major disturbances. It takes most of the circumnatant species, while it seems that some extranatant species might escape (some Canthidermis maculata and Elagatis bipinnulata were observed with recent skin scarring that were certainly caused by netting, which shows that they escaped the fishing gear) and most intranatant species are not captured. When such a fish aggregation is dramatically modified by fishing, what are the re-colonization processes?

The presence of certain species can play a key role in the presence of others, such as circumnatant species. Fishermen firmly stated that they never observed tuna around FADs when non tuna species are not present (Moreno et al. 2007), which suggests that these non tuna species are likely to play a key role in the attraction or aggregation processes of tuna. For instance, one of the most abundant and ubiquitous species in our study was the spotted oceanic triggerfish Canthidermis maculata which in the equatorial region sometimes formed massive schools of many thousands individuals around the drifting FADs. Though no acoustic recordings have been made of Canthidermis maculata production of drumming sounds has been described in other triggerfish (Balistidae) (Salmon et al. 1968). If Canthidermis maculata is a soniferous species, it could be a key species responsible for the generation of biological sounds at drifting FADs, which could be used by some species to locate them (Kingsford et al. 2002; but see Mann et al. 2007). Studying interspecies dependency relations is of major importance to better understanding the complex community processes occurring at FADs that contribute to catches of hundreds of thousands of tons of tuna each year.

Acknowledgements. This work was co-funded by the E.U. Fadio Project (DG Research - QLRI-CT- 2002-02773) and the E.U. Dorade project (DIRED-IFREMER n ${ }^{\circ} 31008 / \mathrm{DIRED} / \mathrm{JPP} / \mathrm{rp}$ ) with the additional support of the Regional Council of Reunion Island. We are grateful to the whole scientific team of the FADIO and DORADE projects, to the crews of the M/V Indian Ocean Explorer and Cap Morgan fishing vessel. Many thanks to the French and Spanish skippers of the purse-seine fishing fleet for their collaboration on their drifting FADs.

\section{References}

Andaloro F, Sinopoli M, Coen B, Potoschi A. 2002, Fish community associated with fads in southern Tyrrhenian Sea and northern Ionian Sea. Biol. Mar. Medit. 9, 236-237.

Bochard R., Techer G., 2004, Écologie alimentaire des thons bananes (Acanthocybium solandri) agrégés autour des objets flottants dans le sud-ouest de l'océan Indien. Mémoire de Maîtrise de biologie des populations et des écosystèmes, Université de La Réunion.

Bohnsack J. A., Bannerot, S. P., 1986, A stationary visual census technique for quantitatively assessing community structure of coral reef fishes. NOAA Tech. Rep. NMFS 41.

Buckley T.W., Miller B.S., 1994, Feeding habits of yellowfin tuna associated with fish aggregating devices in American Samoa. Bull. Mar. Sci. 55, 445-459.

Castro J.J., Santiago J.A., Santana-Ortega, A.T., 2002, A general theory on fish aggregation to floating objects: an alternative to the meeting point hypothesis. Rev. Fish. Biol. Fish. 11, 255-277.

Colvocoresses J., Acosta A., 2007, A large-scale field comparison of strip transect and stationary point count methods for conducting length-based underwater visual surveys of reef fish populations. Fish. Res. 85, 130-141.

D’Anna G., Badalamenti F., Riggio S., 1999a, Traditional and experimental floating fish aggregating devices in the Gulf of Castellammare (NW Sicily): Results from catches and visual observations. Sci. Mar. 63, 209-218.

D’Anna G., Lipari R., Badalamenti F., Cuttitta A., 1999b, Questions arising from the use of visual census in natural and artificial habitats. In: Vacchi M. (eds.), Fish Visual Census in Marine Protected Areas. Il Naturalista Siciliano 23, 187-204.

Dagorn L., Holland K.N., Itano D.G., 2007, Behaviour of yellowfin (Thunnus albacares) and bigeye (T. obesus) tuna in a network of Fish Aggregating Devices (FADs). Mar. Biol. 151, 595-606.

Dempster T., 2003, Association of pelagic fish with floating structures: patterns, processes and ecological consequences. $\mathrm{PhD}$ Thesis, University of Sydney.

Dempster T., Kingsford M.J., 2003, Homing of pelagic fish to fish aggregating devices (FADs): an investigation of the role of sensory cues. Mar. Ecol. Prog. Ser. 258, 213-222.

Dempster T., Taquet M., 2004, Analysis of the literature on fish aggregation devices (FAD) research; gaps in current knowledge and future research directions for studies of FAD-associated fish. Rev. Fish. Biol. Fish. 14, 21-42.

Fabi G., Grati F., Lucchetti A., Trovarelli L., 2002, Evolution of the fish assemblage around a gas platform in the northern Adriatic Sea. ICES J. Mar. Sci. 59, 309-315.

Fabi G., Grati F., Puletti M., Scarcella G., 2004, Effects on fish community induced by installation of two gas platforms in the Adriatic Sea. Mar. Ecol. Prog. Ser. 273, 187-197.

Fonteneau A., Pallares P., Pianet R., 2000, A worldwide review of purse seine fisheries on FADs. In: Le Gall J.-Y., Cayré P., Taquet M. (eds.), Pêche thonière et dispositifs de concentration de poissons. Ed. Ifremer, Actes Colloques 28, 15-34.

Freon P., Dagorn L., 2000, Review of fish associative behaviour: toward a generalisation of the meeting point hypothesis. Rev. Fish. Biol. Fish. 10, 183-207. 
Graham N., Evans R., Russ G., 2003, The effects of marine reserve protection on the trophic relationships of reef fishes on the Great Barrier Reef. Environ. Conserv. 30, 200-208.

Graham N., McClanahan T., Letourneur Y., Galzin R., 2006, Anthropogenic stressors, inter-specific competition and ENSO effects on a Mauritian coral reef. Environ. Biol. Fish. 78, 57-69.

Hall M., Lennert-Cody C. Garcia M., Arenas P., 1999, Characteristics of floating objects and their attractiveness for tunas. In: Scott M.D., Bayliff W. H., Lennert-Cody C.E., Schaefer K.M. (eds.), Proc. Int. Workshop on the Ecology and Fisheries for Tunas Associated with Floating Objects, February 11-13, 1992. InterAm. Trop. Tuna Comm. Spec. Rep. 11, La Jolla, California, pp. 396-446.

Harmelin-Vivien M., Harmelin J.G., Chauvet C., Duval C., Galzin R., Lejeune P., Barnabé G. Blanc F., Chevalier R., Duclerc J., Lasserre G., 1985, Evaluation visuelle des peuplements et populations de poissons : méthodes et problèmes. Rev. Ecol. (TerreVie) 40, 467-539.

Jones J., 1772, Oppian's Halieuticks of the Nature of Fishes and Fishing of the Ancients in V. Books. Translated from the Greek by J. Jones, with an account of Oppian's Life \& Writings, and a Catalogue of his Fishes, Oxford.

Kingsford M., Leis J., Shanks A., Lindeman K., Morgan S., Pineda J., 2002, Sensory environments, larval abilities and local selfrecruitment. Bull. Mar. Sci. 70, 341-375.

Lecchini D., Nakamura Y., Tsuchiya M., Galzin R., 2007, Spatiotemporal distribution of nocturnal coral reef fish juveniles in Moorea Island, French Polynesia. Ichthyol. Res. 54, 18-23.

Lennert-Cody C.E., Hall M.A., 2000, The development of the purse seine fishery on drifting aggregating devices in the Eastern Pacific Ocean: 1992-1998. In: Le Gall J.-Y., Cayré P., Taquet M. (eds.), Pêche thonière et dispositifs de concentration de poissons. Ed. Ifremer, Actes de Colloques 28, 78-107.

Mann D., Casper B., Boyle K., Tricas T., 2007, On the attraction of larval fishes to reef sounds. Mar. Ecol. Prog. Ser. 338, 307-310.

Ménard F., Stéquert B., Rubin A., Herrera M., Marchal E., 2000, Food consumption of tuna in the Equatorial Atlantic Ocean: FADassociated versus unassociated schools. Aquat. Living Resour. 13, 233-240.

Moreno G., Dagorn L., Sancho G., Itano D., 2007, Fish behaviour from fishers' knowledge: the case study of tropical tuna around drifting fish aggregating devices (DFADs). Can. J. Fish. Aquat. Sci. 64, 1517-1528.

Morales-Nin B., Cannizzaro L., Massuti E., Potoschi A., Andaloro F., 2000, An overview of the FADs fishery in the Mediterranean Sea. In: Le Gall J.-Y., Cayré P., Taquet M. (eds.), Pêche thonière et dispositifs de concentration de poisons. Ed. Ifremer, Actes de Colloques 28, pp. 184-207.

Olson R.J., Galvan-Magana F., 2002, Food habits and consumption rates of common dolphinfish (Coryphaena hippurus) in the eastern Pacific Ocean. Fish. Bull. 100, 279-298.

Oxenford H.A., 1999, Biology of the dolphin-fish (Coryphaena hippurus) in the western central Atlantic. Sci. Mar. 63, 277-301.

Parin N.V., Fedoryako B.I., 1999, Pelagic fish communities around floating objects in the open ocean. In: Scott M.D., Bayliff W.H., Lennert-Cody C.E. Schaefer K.M. (eds.), Proc. Int. Workshop on the Ecology and Fisheries for Tunas Associated with Floating Objects, February 11-13, 1992. Inter-Am. Trop. Tuna Comm. Spec. Rep. 11, La Jolla, California, pp. 447-458.

Rilov G, Benayahu Y., 2000, Fish assemblage on natural versus vertical artificial reefs: The rehabilitation perspective. Mar. Biol. 136, 931-942.

Romanov E.V., 2002, By-catch in the tuna purse-seine fisheries of the Western Indian Ocean. Fish. Bull. 100, 90-105.

Salmon M., Winn H.E., Sorgente N., 1968, Sound production and acoustical behavior in triggerfish. Pacif. Sci. 12, 11-20

Takahashi M., Urakawa T., Kasahara F., Kanda A., 1988, JAMARC's activities on tagging on tunas in Indian Ocean. Expert consultation on stock assessments of tunas in the Indian Ocean, Mauritius, 22-27 June 1988. IPTP Collect. Vol. Work. Doc. 3, pp. 208-223.

Taquet M., 2004, Le comportement agrégatif de la dorade coryphène (Coryphaena hippurus) autour des objets flottants. Thèse de Doctorat, Université de Paris 6, Océanologie biologique.

Taquet M., Diringer A., 2007, Guide des poissons de l'océan Indien et de la mer Rouge. Editions Quae, Versailles.

Tessier E., Chabanet P., Pothin K., Soria M., Lasserre G, 2005, Visual censuses of tropical fish aggregations on artificial reefs: slate versus video recording techniques. J. Exp. Mar. Biol. Ecol. 315, $17-$ 30 . 